\title{
Dynamic contrast enhanced-MRI and diffusion-weighted image as predictors of lymphovascular invasion in node-negative invasive breast cancer
}

\author{
Bo Bae Choi®
}

\begin{abstract}
Background: Lymphovascular invasion (LVI) is an important risk factor for prognosis of breast cancer and an unfavorable prognostic factor in node-negative invasive breast cancer patients. The purpose of this study was to evaluate the association between LVI and pre-operative features of dynamic contrast-enhanced magnetic resonance imaging (DCE-MRI) and diffusion-weighted imaging (DWI) in node-negative invasive breast cancer.

Methods: Data were collected retrospectively from 132 cases who had undergone pre-operative MRI and had invasive breast carcinoma confirmed on the last surgical pathology report. MRI and DWI data were analyzed for the size of tumor, mass shape, margin, internal enhancement pattern, kinetic enhancement curve, high intratumoral T2weighted signal intensity, peritumoral edema, DWI rim sign, and apparent diffusion coefficient (ADC) values. We calculated the relationship between presence of LVI and various prognostic factors and MRI features.

Results: Pathologic tumor size, mass margin, internal enhancement pattern, kinetic enhancement curve, DWI rim sign, and the difference between maximum and minimum ADC were significantly correlated with LVI $(p<0.05)$.

Conclusions: We suggest that DCE-MRI with DWI would assist in predicting LVI status in node-negative invasive breast cancer patients.
\end{abstract}

Keywords: Breast cancer, Magnetic resonance imaging, Diffusion-weighted imaging, Lymphovascular invasion

\section{Background}

Prognostic factors are important for the management and treatment of breast cancer. Classical prognostic factors are axillary lymph node (ALN) status, tumor size, and nuclear and histological grade, while estrogen receptor (ER), progesterone receptor (PR), and human epidermal growth factor 2 (HER2) are known as molecular prognostic factors $[1,2]$. Lymphovascular invasion (LVI) is defined as the presence of tumor cells within a definite endothelial-lined space (lymphatic or blood vascular

Correspondence: med20@cnuh.co.kr

Department of Radiology, Chungnam National University Hospital, 282

Munhwa-ro, Jung-gu, Daejeon 35015, Republic of Korea system) in the breast surrounding invasive carcinoma [3]. The presence of LVI is associated with an increased risk of ALN and distant metastases [4], and is an unfavorable prognostic indicator for breast cancer survival and recurrence [5-7]. In particular, in patients with node-negative invasive breast cancer LVI is an independent prognostic factor for survival, recurrence, and distant metastasis [8]. The presence of LVI is currently determined at surgery and recorded on the surgical pathology report, but if certain features related to LVI could be detected by pre-operative imaging, predictions on tumor prognosis including lymph node (LN) metastasis could

(c) The Author(s). 2021 Open Access This article is licensed under a Creative Commons Attribution 4.0 International License, which permits use, sharing, adaptation, distribution and reproduction in any medium or format, as long as you give appropriate credit to the original author(s) and the source, provide a link to the Creative Commons licence, and indicate if changes were made. The images or other third party material in this article are included in the article's Creative Commons licence, unless indicated otherwise in a credit line to the material. If material is not included in the article's Creative Commons licence and your intended use is not permitted by statutory regulation or exceeds the permitted use, you will need to obtain permission directly from the copyright holder. To view a copy of this licence, visit http://creativecommons.org/licenses/by/4.0/ The Creative Commons Public Domain Dedication waiver (http://creativecommons.org/publicdomain/zero/1.0/) applies to the data made available in this article, unless otherwise stated in a credit line to the data. 
be made, and choice of chemotherapy could be informed, prior to surgery.

There have been several reports on the association between LVI and magnetic resonance imaging (MRI) parameters [9-11], of them, only a few studies about MRI features with easy access of analysis at practical field. Shi et al. [10] analyzed the correlation of LVI with mass margin, diffusion-weighted imaging (DWI) signal, and time intensity curve, and Cheon et al. [11], in addition included peritumoral edema and adjacent vessel sign in their analysis. However, a few factors showed discordance between the two studies. To achieve a consensus on the MRI parameters that correlate with LVI, more studies with different approaches are needed.

DWI is an MRI technique based on the difference in random motion of water molecules between tissues, and is a very sensitive means of detecting cell density, membrane integrity, and tumor microstructure. The apparent diffusion coefficient (ADC) is a measure of the degree of water diffusion within tissue. DWI and the ADC are effective for discriminating benign and malignant tissue, improving the diagnosis of breast cancer when combined with dynamic contrast-enhanced MRI (DCE-MRI), and they are effective for response monitoring after neoadjuvant chemotherapy $[12,13]$. ADC values have been associated with classical and molecular prognostic factors, as well as treatment response [14-16]. However, the results are discordant, with no standard value identified and limited reproducibility between the different machines, conditions, or protocols used. In studies of LVI and ADC, LVI has been shown to associate with either the medial, minimum, or peritumoral ADC, depending on the study design [17-19]. To improve generalizability and accuracy, we used the ADC difference value (ADCdif), derived by subtracting the minimum ADC of the mass from its maximum. ADC-dif represents the heterogeneity of the tumor and is a parameter associated with several prognostic features of MRI [20, 21]. We also studied the association between DWI rim sign and LVI. The DWI rim sign is the presence of a high intensity signal around the rim of the mass, which was reported to correlate with tumor malignancy by Kang et al. [22].

The purpose of this study was to evaluate the association between various MRI features, including from DWI, and LVI in node-negative invasive breast cancer.

\section{Methods}

\section{Patients}

Data from patients who underwent pre-operative MRI and breast cancer surgery (mastectomy or lumpectomy) for invasive ductal carcinoma (IDC) that was not otherwise specified (NOS) between July 2017 and October 2018 were collected retrospectively from our institutional database. Cases of neo-adjuvant chemotherapy and node-positive on the surgical pathology report were excluded. Finally, 132 cases of IDC NOS type breast cancer reported as node-negative on the surgical pathology report were included in this study.

\section{MRI acquisition techniques and interpretation}

MRI was performed with the patient in the prone position using a $1.5 \mathrm{~T}$ scanner (Signa Excite, GE Healthcare, Milwaukee, WI, USA) equipped with a dedicated 8channel surface breast coil. Images were acquired in the axial plane with the following sequences: axial, T2weighted (T2W), fat-suppressed, fast spin-echo imaging (TR/TE, 5000/86; flip angle, $90^{\circ}$; field of view (FOV), 280-360 mm; acquisition matrix, $320 \times 256$; number of excitations (NEX), 3; slice thickness, $4.5 \mathrm{~mm}$ ) and preand post-contrast, axial, T1-weighted (T1W) 3dimensional (3D) fast spoiled gradient-recalled echo sequence with parallel volume imaging (VIBRANT, GE Healthcare, Oslo, Norway) (TR/TE, 6.6/3.2; flip angle, $10^{\circ}$; FOV, $280-360 \mathrm{~mm}$; acquisition matrix, $360 \times 360$; NEX, 0.8; slice thickness, $1.1 \mathrm{~mm}$ ). Gadodiamide (Omniscan, GE Healthcare) was administered as the contrast agent with an intravenous bolus injection $(0.2$ mmol per $\mathrm{kg}$ body weight) at a rate of $3 \mathrm{ml} / \mathrm{s}$. Imaging was performed before the intravenous contrast agent bolus injection and four times afterwards for a period of $7.3 \mathrm{~min}$. The image post-processing included the subtraction of unenhanced images from enhanced images, sagittal reformations, and 3D maximum-intensity projections using the first contrast-enhanced series. DWI was performed using spin-echo single shot echo-planar imaging with $b$ values of 0 and $1000 \mathrm{~s} / \mathrm{mm}^{2}$; TR/TE, 4000/ $61.6 \mathrm{~ms}$; FOV, $280-360 \mathrm{~mm}$; acquisition matrix, $90 \times$ 128; NEX, 6; slice thickness, $4.5 \mathrm{~mm}$; and gap, $0.6 \mathrm{~mm}$. DWI was performed in each of three orthogonal directions along the $x, y$, and $z$ axes. Images from each direction were combined to produce a single image at each slice location showing the diffusion-restricted area. The signal intensities of all three directions were combined $(\mathrm{Scmb})$ so that $\mathrm{Scmb}=\{\mathrm{Sx} \cdot \mathrm{Sy} \cdot \mathrm{Sz}\} / 3$, where $\mathrm{Sx}, \mathrm{Sy}$, and $\mathrm{Sz}$ are the signal intensity values of the three orthogonal directions. ADC values were calculated according to the following formula: $A D C=[1 /(b 2-b 1)] \ln (S 1 / S 2)$, where $\mathrm{S} 1$ and $\mathrm{S} 2$ are the signal intensities in the regions of interest (ROIs) obtained from the two gradient factors $\mathrm{b} 2$ and $\mathrm{b} 1 \mathrm{~b} 1=0$ and $\mathrm{b} 2=1000 \mathrm{~s} / \mathrm{mm}^{2}$ for the $1.5 \mathrm{~T}$ scanner).

The interpretation of the degree and patterns of enhancement was performed by visual assessment. One breast radiologist (with 10 years of experience) reviewed the breast cancer MRI images. The vertical and horizontal dimensions of the masses were measured on the image section showing the largest mass on earlyenhancement T1W images, and the largest diameter 
among the vertical and horizontal dimensions was recorded. In the case of multifocal and multicentic tumors, the size was measured at the largest mass by the same method. For non-mass enhancement lesions, total extent of the lesion was measured and its size was recorded. MRI was reported using the Breast Imaging Reporting and Data System (BI-RADS) MRI lexicon [23] with respect to mass or non-mass lesion, mass shape (oval, round, irregular), mass margin (circumscribed, irregular, spiculated), and internal enhancement characteristics (homogeneous, heterogeneous, rim enhancement). Rim enhancement was defined as strong enhancement at the periphery of a tumor compared with that at the center. MRI enhancement kinetics were evaluated by visual assessment as follows: type I curve (progressive enhancement pattern), type 2 curve (plateau pattern), and type 3 curve (washout pattern). A type 2 curve was not observed in this study. Intratumoral high signal intensity on T2W images was assessed compared with surrounding normal breast parenchymal signal intensity on fatsuppressed T2W images, and was defined as very high signal (when the signal was similar to a water or vessel signal), high signal (when the mass signal was higher than that of surrounding breast tissue), and low signal (when the mass signal was lower than that of surrounding breast tissue). Peritumoral edema was assessed by visual assessment of fat-suppressed T2W images. Peritumoral edema was recorded as present when a high signal was noted posterior to the tumor mass in the prepectoral area, or when there was a fairly extensive high signal around the tumor mass. The ADC of a mass was measured by manually placing ROIs within a mass on the ADC maps automatically calculated by the MRI software. Axial ADC maps representing the largest diameter of the mass were selected for ROI placements. Multiple ROIs of $17-23 \mathrm{~mm}^{2}$ were placed within the mass, with as many as possible being examined [24] (Fig. 1c). ROIs were carefully placed to avoid cystic areas, necrotic areas, and visual DWI artifacts. The lowest ADC value from the multiple ROIs was regarded as the minimum $\mathrm{ADC}$, and the highest $\mathrm{ADC}$ value as the maximum ADC. The difference between the maximum and minimum ADC was recorded as ADC-dif. A high signal rim surrounding a mass was recorded as a positive DWI rim sign, irrespective of whether it was complete or incomplete [22].

\section{Histologic analysis}

Pathological reports from core needle, excisional biopsies, breast-conserving surgery, or mastectomy specimen were reviewed by two breast pathologists (with 20 and 5 years of experience). Histological analysis was carried out on specimens obtained at the last surgery. Pathologists reported tumor histologic type, invasive tumor size, and LN status. LVI was assessed on hematoxylin and eosin-stained sections, and was defined as carcinoma cells in a definite endothelial-lined space in the peritumoral breast surrounding the invasive carcinoma. Immunohistochemical staining was used to determine the expression of the following molecular markers: ER, PR, HER2, and Ki-67. ER and PR expression were quantified using the Allred scoring system, and were considered as positive for ER or PR with a total Allred score $>2$ [25]. The intensity of HER2 staining was scored as $0,1+, 2+$, or $3+$. Tumors with a score of $3+$ were classified as HER2 positive, while tumors with a score of 0 or $1+$ were negative. A HER2 value of 2 was considered equivocal. For equivocal result, further testing of fluorescence in situ hybridization (FISH) was conducted, and pathologists reported the final molecular types of breast cancer. Ki-67 expression was recorded as the percentage of tumor epithelial cells determined by immunohistochemical assay, both digital image analysis and visual scoring by light microscope were used for quantification.

\section{Statistical analysis}

Analysis of variance was performed for tumor histologic grade, expression of Ki-67, hormone receptor status, existence of LVI, and other MRI findings. For the examination of statistical differences in the clinicopathological variables and MRI features among the LVI positive (LVI+) group and LVI negative (LVI-) group, the chisquare test or Fisher's exact test was used, and the Kruskal-Wallis test was used for histologic grade. The odds ratio (OR) and 95\% confidence interval (CI) for LVI were calculated with univariate logistic regression analysis. The Kolmogorov-Smirnov test was used to test for normality in the analysis of numerical data. The numerical data except for ADC-dif were verified as being normally distributed and were analyzed using the independent sample $t$ test. For ADC-dif, the Mann-Whitney $U$ test was used. A $p$ value of $<0.05$ was considered statistically significant. Statistical analyses were performed using PASW Statistics 18.0 (IBM, Armonk, NY, USA).

\section{Results \\ Patients}

The mean age of 132 cases was 54 years (range, 21-82 years). Mean size of pathologic invasive cancer was 1.8 $\mathrm{cm}$ (range, 0.1-5.2 cm). The most common molecular subtype was ER+ (93/132, 70.5\%), and moderate differentiated was the most common histologic grade (48/132, $36.4 \%)$.

\section{LVI vs. clinicopathological features}

Among 132 cases of IDC NOS, 42 were LVI+ and 90 were LVI- (mean age 53 vs. 54 years, respectively). Analysis of the relationship between LVI and clinicopathologic 

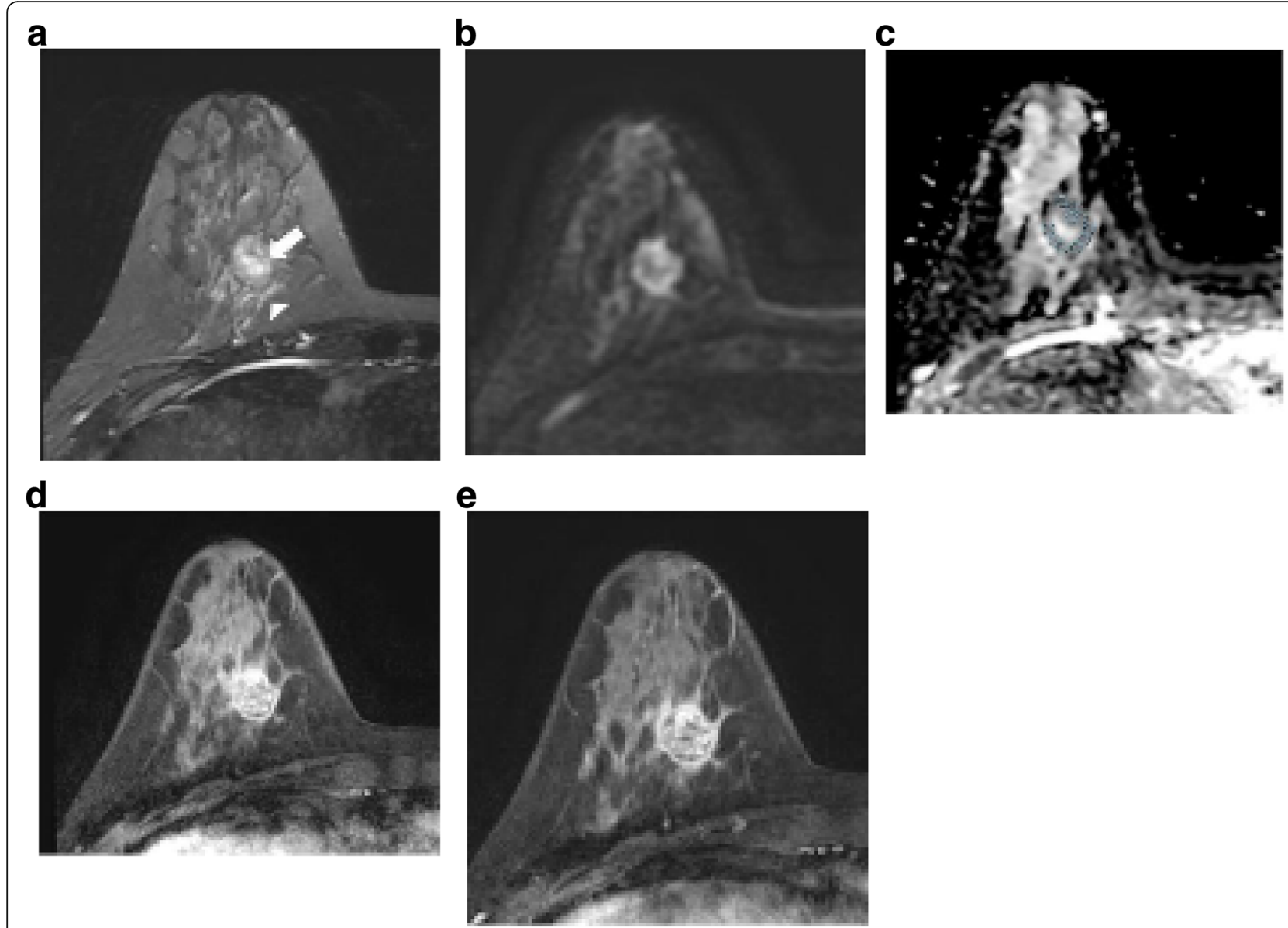

Fig. 1 Images of a 37-year-old woman with invasive ductal carcinoma and a lymphovascular invasion. a Axial T2 weighted image shows a $2.3 \mathrm{~cm}$ mass in her right breast. Intratumoral T2 high signal (arrow) and peritumoral edema (arrowhead) are noted. $\mathbf{b}$ High signal intensity rim is shown in axial diffusion weighted image ( $b$ value $=1000 \mathrm{~s} / \mathrm{mm}^{2}$ ). c Apparent diffusion coefficient map shows restriction along the periphery of the mass. Multiple region of interests (ROI)s of $6.87 \mathrm{~mm}^{2}$ were manually placed within the mass avoiding cystic or necrotic area. Minimum ADC value was $1025 \times 10^{-6} \mathrm{~mm}^{2} / \mathrm{s}$, and maximum ADC value was $1345 \times 10^{-6} \mathrm{~mm}^{2} / \mathrm{s}$. The calculated ADC difference was $320 \times 10^{-6} \mathrm{~mm}^{2} / \mathrm{s}$. d, e Axial contrastenhanced T1-weighted image after 2 min (d) and 6 min (e) of contrast injection demonstrating a round, circumscribed mass in right breast. This mass shows rim internal enhancement and washout kinetic pattern of enhancement. This patient underwent modified radical mastectomy of her right breast. The histopathological features of this mass were poorly differentiated, no lymph node metastasis, ER-. PR-, HER2- and Ki-67 high (70\%). Tumor size at final pathologic report was $3.6 \mathrm{~cm}$

prognostic factors revealed a significant correlation between invasive tumor size (both as measured by surgical specimen and MRI) and LVI (Table 1). Histologic grade, Ki-67, and hormone receptor status showed no correlation with LVI.

\section{LVI vs. MRI features}

We found significant associations between LVI and mass margin, internal enhancement pattern, and outcome of the kinetic enhancement curve analysis (Table 2). For the LVI+ group, circumscribed, irregular, or spiculated margins occurred to similar degrees, while a circumscribed margin was the most common in the LVIgroup (Figs. 1 and 2). A rim enhancement pattern of the mass was most common in the LVI+ group (Fig. 1d), while in the LVI- group it was the homogeneous pattern
(Fig. 2c). In the enhancement curve analysis, a washout pattern was most common in both the LVI groups (Figs. 1 and 2), but the washout pattern showed more in the $\mathrm{LVI}+$ group ( $85.7 \%$ vs. $66.7 \%)$. A high intratumoral T2W signal and peritumoral edema were equally uncommon in both the LVI groups (Fig. 1a), and there was no correlation with neither factor nor LVI.

Analysis of the relationship between LVI and DWI findings revealed that the LVI+ group had a significantly higher incidence of the DWI rim sign than the LVIgroup (Fig. 1b). Of the different types of ADC values analyzed vs. LVI, only ADC-dif was significantly correlated with LVI ( $p=0.000$, Figs. 1 and 2). The maximum ADC value was higher in the LVI+ group, but was not significantly different from than in the LVI- group $(p=0.059$; Tables 2 and 3 ). 
Table 1 Clinicopathological characteristics of patients according to lymphovascular invasion status

\begin{tabular}{|c|c|c|c|c|}
\hline Variables & $\mathrm{LVI}+(n=42)$ & LVI- $(n=90)$ & OR & $P$ value \\
\hline Mean age (years) ${ }^{a}$ & $53 \pm 1.75$ & $54 \pm 1.20$ & & 0.494 \\
\hline Histologic grade & & & $0.7(0.4-1.1)$ & $0.080^{b}$ \\
\hline Well & $7(16.7)$ & 32 (35.6) & & \\
\hline Moderate & $19(45.2)$ & $29(32.2)$ & & \\
\hline Poor & $16(38.1)$ & $29(32.2)$ & & \\
\hline Ki-67 status & & & $1.0(0.5-2.1)$ & 0.986 \\
\hline High ( $\geq 14 \%)$ & $22(52.4)$ & $47(52.2)$ & & \\
\hline Low (<14\%) & $20(47.6)$ & $42(47.8)$ & & \\
\hline Tumor subtype & & & $0.9(0.6-1.5)$ & 0.873 \\
\hline ER-positive & $29(69)$ & $64(71.1)$ & & \\
\hline HER2-positive & $5(11.9)$ & $12(13.3)$ & & \\
\hline Triple-negative & $8(19)$ & $14(15.6)$ & & \\
\hline Pathologic tumor size $(\mathrm{cm})$ & $2.1 \pm 0.13$ & $1.6 \pm 0.08$ & $0.5(0.3-0.7)$ & $0.001^{c}$ \\
\hline MRI tumor size & $2.0 \pm 0.15$ & $1.7 \pm 0.11$ & $0.8(0.5-1.1)$ & 0.007 \\
\hline
\end{tabular}

ER Estrogen receptor, HER2 Human epithermal growth factor receptor 2

*Data are numbers of patients, with percentages in parentheses unless otherwise indicated

${ }^{a}$ Data are mean age of patients

bruskal-Wallis test

'Mann-Whitney $U$ test

Table 2 MRI features and lymphovascular invasion status in node-negative breast cancer patients

\begin{tabular}{|c|c|c|c|c|}
\hline Variables & $\mathrm{LVI}+(n=42)$ & LVI- $(n=90)$ & OR & $P$ value \\
\hline Mass shape & & & $0.6(0.3-1.3)$ & 0.169 \\
\hline Oval or round & $17(40.5)$ & $48(53.3)$ & & \\
\hline Irregular & $25(59.5)$ & $42(46.7)$ & & \\
\hline Mass margin & & & $0.6(0.3-0.9)$ & 0.045 \\
\hline Circumscribed & $15(35.7)$ & $46(51.1)$ & & \\
\hline Irregular & $14(33.3)$ & $32(35.6)$ & & \\
\hline Spiculated & $13(31.0)$ & $12(13.3)$ & & \\
\hline Mass internal enhancement & & & $0.5(0.3-0.8)$ & 0.007 \\
\hline Homogeneous & $6(14.3)$ & $37(41.1)$ & & \\
\hline Heterogeneous & $16(38.1)$ & $28(31.1)$ & & \\
\hline $\operatorname{Rim}$ & $20(47.6)$ & $25(27.8)$ & & \\
\hline Kinetic analysis & & & $0.3(0.1-0.9)$ & 0.022 \\
\hline Progressive pattern & $6(14.3)$ & $30(33.3)$ & & \\
\hline Washout pattern & $36(85.7)$ & $60(66.7)$ & & \\
\hline Intratumoral T2 high signal & & & $2.8(0.9-8.3)$ & $0.077^{a}$ \\
\hline Positive & $8(19.0)$ & $7(7.8)$ & & \\
\hline Negative & $34(81.0)$ & $83(92.2)$ & & \\
\hline Peritumoral edema & & & $1.6(0.6-3.8)$ & 0.329 \\
\hline Positive & $10(23.8)$ & $15(16.7)$ & & \\
\hline Negative & $32(76.2)$ & $76(83.3)$ & & \\
\hline DWI rim sign & & & $2.5(1.2-5.4)$ & 0.017 \\
\hline Positive & $28(66.7)$ & $40(44.4)$ & & \\
\hline Negative & $14(33.3)$ & $50(55.6)$ & & \\
\hline
\end{tabular}

DWI Diffusion weighted image

*Data are numbers of patients, with percentages in parentheses unless otherwise indicated

${ }^{\mathrm{a}}$ Fisher's exact test 

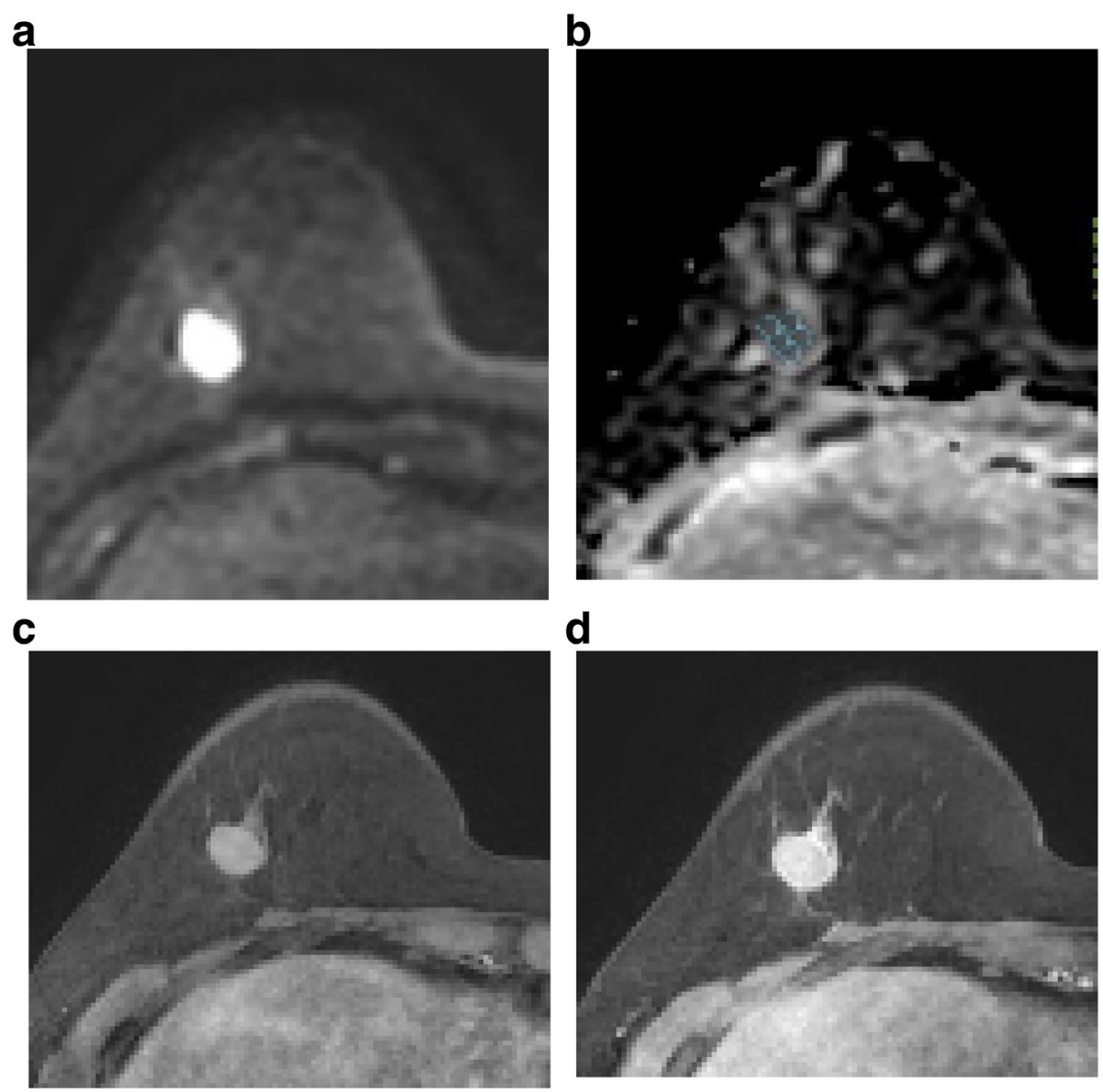

Fig. 2 Images of a 59-year-old woman with invasive ductal carcinoma without lymphovascular invasion. a Diffusion-weighted image demonstrates homogeneous high signal intensity of mass without rim sign ( $b$ value $=1000 \mathrm{~s} / \mathrm{mm}^{2}$ ). b Apparent diffusion coefficient map. Multiple region of interests (ROI)s of $7.81 \mathrm{~mm}^{2}$ were manually placed within the mass avoiding cystic or necrotic area. Minimum ADC value was $826 \times$ $10^{-6} \mathrm{~mm}^{2} / \mathrm{s}$, and maximum ADC value was $1069 \times 10^{-6} \mathrm{~mm}^{2} / \mathrm{s}$. The calculated ADC difference was $243 \times 10^{-6} \mathrm{~mm}^{2} / \mathrm{s}$. c, d Axial contrastenhanced T1-weighted image after $2 \mathrm{~min}$ (c) and $6 \mathrm{~min}$ (d) of contrast injection demonstrating an oval, circumscribed mass with homogeneous enhancement in her right breast. Kinetic analysis of enhancement pattern was washout pattern. This patient underwent modified radical mastectomy. The histopathological features of this mass were moderate differentiated, no lymph node metastasis, ER-, PR-, HER2+, and Ki-67 high (20\%). Tumor size at final pathologic report was $1.6 \mathrm{~cm}$

\section{Discussion}

LVI is a risk factor for ALN and distant metastasis, and helps to predict survival and local or distant recurrence in node-negative invasive breast cancer patients [4-8]. LVI status is also effective for response evaluation after neo-adjuvant chemotherapy (NAC) [3]. Previous studies have reported a correlation between LVI and wellknown prognostic factors of breast cancer, including hormone receptor expression status, tumor size, ALN status, age, and histological grade $[14,26]$, but the results between studies are discordant [11]. In the present study, invasive tumor size was related to LVI; however, we found no correlation with histologic grade, hormone receptor status, or Ki-67 status.

LVI was correlation with morphologic and kinetic features of breast cancer on DCE-MRI. We found

Table 3 Quantitative ADC parameters according to lymphovascular invasion status

\begin{tabular}{llll}
\hline Variable & LVI+ $(\boldsymbol{n}=\mathbf{4 2})$ & LVI- $(\boldsymbol{n}=\mathbf{9 0})$ & \\
\hline Minimum ADC $\left(\times 10^{-3} \mathrm{~mm}^{2} / \mathrm{s}\right)$ & $0.917 \pm 0.023$ & $0.931 \pm 0.020$ & 0.674 \\
Maximum ADC $\left(\times 10^{-3} \mathrm{~mm}^{2} / \mathrm{s}\right)$ & $1.163 \pm 0.216$ & $1.092 \pm 0.190$ & 0.059 \\
Mean ADC $\left(\times 10^{-3} \mathrm{~mm}^{2} / \mathrm{s}\right)$ & $1.040 \pm 0.174$ & $1.011 \pm 0.179$ & 0.393 \\
ADC difference ADC $\left(\times 10^{-3} \mathrm{~mm}^{2} / \mathrm{s}\right)$ & $0.246 \pm 0.134$ & $0.162 \pm 0.111$ & 0.0 \\
\hline
\end{tabular}


significant correlation between LVI and mass margin, internal enhancement pattern, and type of kinetic enhancement curve. And, this result is the novelty of our study. Shi et al. [10] reported that LVI cases showed an ill-defined margin and lobulation. Although those specific terms are not included in the BI-RADS categories, ill-defined margin and lobulation can be considered as equating to the BI-RADS terminology "not-circumscribed margin." In our study, the LVI+ cases had a higher incidence of an irregular and spiculated margin than the LVI-, which concurs with the findings of Shi et al. [10]. Cancer induces a desmoplastic reaction in adjacent tissue with growing, and irregular or spiculated margins are noted on imaging $[27,28]$. In rapidly growing aggressive cancers such as triple negative breast cancer (TNBC), the desmoplastic reaction cannot catch up with the tumor growing, resulting in a circumscribed margin visible on imaging [29]. However, the mass margin alone is not sufficient to evaluate the aggressiveness of a tumor.

Washout pattern was the most common type of kinetic enhancement curve in both LVI+ and LVI- cases, with more percentages on LVI+ $(85.7 \%$ in $\mathrm{LVI}+$ vs. $66.7 \%$ in LVI-) $(p=0.022)$. This result correlates with those of Shi et al. [10], showing a significant association between the presence of LVI and a plateau or washout pattern. We found an association between the presence of LVI and a heterogeneous or rim enhancement pattern (85.7\%, $p=0.007)$, while Cheon et al. [11] reported no correlation between the internal enhancement pattern and LVI. A washout kinetic curve pattern or rim enhancement pattern in breast cancer are reported as prognostic factors, and are associated with higher histologic grade and negative ER status, as well as with TNBC [30-33]. Washout pattern is not usually seen in benign breast masses (specificity, 90.4\%) and is considered suggestive of a malignant mass [30]. The relationship between kinetic parameters and prognostic factors is controversial; however, a significant correlation has been reported between washout curve pattern and higher histologic grade, Ki-67 positivity, and negative ER status [31]. Rim enhancement is defined as enhancement more obvious at the periphery of the mass, whether it has a thin or thick rim pattern, and has been shown to correlate with histologic grade, size, and LN status [31]. Our present study showed that the significant association between the presence of LVI and MRI feature of washout curve pattern, rim enhancement, which is helpful for prediction analysis of breast cancer prognosis with DCEMRI.

Several studies have shown that ADC values were associated with LVI, although, among multiple ADC parameters, optimal ADC parameters to match the study results varied with study design [17-19]. In studies of the relationship between ADC and LVI, Yang et al. [19] report that the minimum ADC correlates with LVI, while Karan et al. [17] state that the median ADC associates with LVI. Our study found no significant correlation for LVI with either the minimum, maximum, or mean ADC values (only a borderline correlation was found with maximum $\operatorname{ADC}(p=0.059))$. Besides, the way of drawing the ROI is not always the same, which makes less reproducibility. The reason for the discordance between our results and those of others is likely to be the lack of a standard value for discriminating benign from malignant tissue, and also that there is no standard method for measuring the ROI. Depending on the measuring method used, it is possible for non-invasive tissue to be included in the ROI. To overcome this problem, various measurement methods using different aspects of the ADC have been tried, for example, by applying the values for relative $\mathrm{ADC}$, peritumoral $\mathrm{ADC}$, and $\mathrm{ADC}$-dif [20, 24]. Mori et al. [18] used peritumoral ADC and reported that it is effective in predicting LVI in nodenegative breast cancer.

Breast cancer lesions are very heterogeneous, and understanding tumor heterogeneity is essential. Intratumoral heterogeneity, the presence of heterogeneous cell populations within a tumor, is an indicator for metastatic potential and treatment resistance [32, 33]. Hence, to gain more insight into tumor composition, in our study we used ADC-dif, which can provide a more nuanced reflection of the tumor's characteristics. ADC-dif, the difference between the maximum and minimum ADC, can highlight heterogeneity in the intratumoral cellularity. ADC-dif in combination with the minimum ADC can improve the rate of concordance with histological tumor grading and raise the diagnostic performance of breast MRI [21]. In our study, ADC-dif was significantly higher in LVI+ cases $(p=0.0)$. This result implies that the LVI+ group has more intratumoral heterogeneity than the LVI- group. Therefore, in many cases, especially of pre-operative image evaluations in node-negative patients or when it is difficult to identify LN metastasis with certainty, identifying signs of intratumoral heterogeneity by ADC-dif could be a crucial indicator for further evaluation and treatment of breast cancer.

The DWI rim sign is described by Kang et al. [22] as a high signal intensity rim on DWI outlining $\geq 90 \%$ (complete) or $\leq 90 \%$ (incomplete) of the lesion. Rim sign negativity is defined as no visible high signal on the rim of the lesion on DWI. The DWI rim sign on breast lesions is associated with malignancy [22, 34], but there have been no studies on the influence of the DWI rim sign on prognosis. Choi et al. [34] hypothesized that as DWI rim sign reflects higher cellularity, its presence would vary with breast cancer subtypes, but they found 
no significant difference in DWI rim sign between two breast cancer subtypes. In our study, $66.7 \%$ of LVI+ cases showed the DWI rim sign compared with $44.4 \%$ of LVI- cases, suggesting that DWI rim sign is an important prognostic indicator. We, therefore, analyzed correlations between DWI rim sign and histologic grade, Ki67, pathologic invasive tumor size, and tumor subtype. The DWI rim sign was significantly correlated with histologic grade, pathologic invasive tumor size, and tumor subtype (ER+ or ER-), and showed borderline correlation with Ki-67 (Table 4). Although we studied only node-negative breast cancer, the DWI rim sign would have a strong relationship with prognostic factors of any cancer.

Peritumoral edema and high intratumoral T2W signal on MRI are prognostic factors associated with worse recurrence-free survival [35, 36]. A few studies have demonstrated the association between LVI and peritumoral edema in breast cancer [11, 37]. Peritumoral edema is due to increased vascular permeability and peritumoral cytokines [38], and is a biomarker of aggressive breast cancer, positively associated with larger tumor size, high histologic grade, high Ki-67 value, more metastatic LNs, and recurrence $[39,40]$. In the present study, we found no correlation between LVI and peritumoral edema. Similarly, Mori et al. [18] reported no association between LVI and peritumoral edema, but they did find a correlation between peritumoral ADC and LVI. To measure peritumoral ADC value, ROIs were placed where the ADC values visually appeared to be most increased adjacent to the tumor border [18].
Aggressive breast cancers have higher peritumoral ADC values, reflecting peritumoral edema and increased cell density caused by proliferative changes [41]. Thus, peritumoral ADC might be a more sensitive means of detecting early edema than signs seen on DCE-MRI. High intratumoral T2W signal is not common finding, but is associated with TNBC (25-48\%) [42]. This finding correlates with a higher maximum standard unit value on positron emission tomography-computed tomography [43]. In our study, there was no correlation between LVI and intratumoral T2 high signal. Since this study is only for LN negative patients, we will get better results with larger study encompassing a range of all $\mathrm{LN}+$ and $\mathrm{LN}-$ patients.

This study has several limitations. First, its retrospective nature and data review by only one radiologist at a single institution. The results would be more convincing if the MRI data could be reviewed by more than one radiologist. Second, drawing ROIs on very small tumors $(<0.5 \mathrm{~cm})$ and making multiple ROI measurements of them was difficult. The difficulty in drawing very small ROIs on images retrieved from the picture archiving and communication (PACS) system is a technical drawback of the system; thus, more molecular or biological assessment is needed for very small cancers. Third, this study targeted node-negative invasive breast cancer. Certain factors cannot be generalized to our result and need more generalized data for more accurate outcomes. Forth, it is known that the diagnostic performance of the kinetic analysis of NME in MRI is not as good as that of the mass [44-46]. In this study, there were only 4 cases

Table 4 Clinicopathologic prognostic factors according to DWI rim sign

\begin{tabular}{|c|c|c|c|}
\hline Variables & DWI rim sign $+(n=68)$ & DWI rim sign $-(n=64)$ & $P$ value \\
\hline Histologic grade & & & $0.005^{\mathrm{a}}$ \\
\hline Well & $14(20.6)$ & $25(39.1)$ & \\
\hline Moderate & $24(35.3)$ & $24(37.5)$ & \\
\hline Poor & $30(44.1)$ & $15(23.4)$ & \\
\hline Ki-67 status & & & 0.057 \\
\hline High ( $\geq 14 \%)$ & $41(60.3)$ & $28(43.8)$ & \\
\hline Low (<14\%) & $27(39.7)$ & $36(56.3)$ & \\
\hline Tumor subtype & & & 0.054 \\
\hline ER-positive & $42(61.8)$ & $51(79.7)$ & \\
\hline HER2-positive & $10(14.7)$ & $7(10.9)$ & \\
\hline Triple-negative & $16(23.5)$ & $6(9.4)$ & \\
\hline Tumor subtype & & & 0.024 \\
\hline ER-positive & $42(61.8)$ & $51(79.7)$ & \\
\hline ER-negative & $26(38.2)$ & $13(20.3)$ & \\
\hline Pathologic tumor size $(\mathrm{cm})$ & $2.1 \pm 0.10$ & $1.4 \pm 0.89$ & 0.0 \\
\hline
\end{tabular}

DWI Diffusion weighted image, ER Estrogen receptor, HER2 Human epithermal growth factor receptor 2

*Data are numbers of patients, with percentages in parentheses unless otherwise indicated

${ }^{a}$ Kruskal-Wallis test 
of NME, so comparative analysis of NME and mass was not performed. The reason there are few NME cases is probably because IDC is the only target. If a study including all DCIS, ILC, and other types of breast cancer is conducted in the future, and NME cases are sufficiently included, a detailed analysis will be possible.

\section{Conclusions}

We found that in node-negative invasive breast cancer, LVI was associated with various clinicopathologic prognostic factors and MRI features. DWI with DCE-MRI is helpful in predicting LVI.

\section{Acknowledgements \\ None}

\section{Human and animal rights}

Not applicable

\section{Author's contributions}

SI Choi performed the histological examination of the breast, and was a major contributor in writing the manuscript. All authors read and approved the final manuscript.

\section{Funding}

None

\section{Availability of data and materials}

Data of this article is collected by our institutional own research program, which is limited to our institution.

\section{Declarations}

Ethics approval and consent to participate

Ethical approval was not sought for the present study because this article involves the analysis of existing data.

\section{Consent for publication}

Not applicable

\section{Competing interests}

The authors declare no conflict of interest, financial, or otherwise.

Received: 17 November 2020 Accepted: 9 March 2021

Published online: 15 March 2021

\section{References}

1. Lee SB, Kim J, Sohn G, Kim J, Chung IY, Kim HJ, Ko BS, Son BH, Ahn SH, Lee $J W$, Jung KH. A nomogram for predicting the oncotype DX recurrence score in women with T1-3NO-1 miMO hormone receptor-positive, human epidermal growth factor 2 (HER2)-negative breast cancer. Cancer Res Treat. 2019:51(3):1073-85. https://doi.org/10.4143/crt.2018.357.

2. Nicolini A, Ferrari P, Duffy MJ. Prognostic and predictive biomarkers in breast cancer: past, present and future. Semin Cancer Biol. 2018;52(Pt 1):5673. https://doi.org/10.1016/j.semcancer.2017.08.010.

3. Ryu YJ, Kang SJ, Cho JS, Yoon JH, Park MH. Lymphovascular invasion can be better than pathologic complete response to predict prognosis in breast cancer treated with neoadjuvant chemotherapy. Medicine (Baltimore). 2018; 97:e11647

4. Ejlertsen B, Jensen MB, Rank F, Rasmussen BB, Christiansen P, Kroman N, Kvistgaard ME, Overgaard M, Toftdahl DB, Mouridsen HT, Danish Breast Cancer Cooperative Group. Population-based study of peritumoral lymphovascular invasion and outcome among patients with operable breast cancer. J Natl Cancer Inst. 2009;101(10):729-35. https://doi.org/10.1 093/jnci/djp090.

5. Liao G-S, Hsu H-M, Chu C-H, Hong Z-J, Fu C-Y, Chou Y-C, et al. Prognostic role of lymphovascular invasion and lymph node status among breast cancer subtypes. J Med Sci. 2018;38:54-61.
6. Paduch $R$. The role of lymphangiogenesis and angiogenesis in tumor metastasis. Cell Oncol (Dordr). 2016;39(5):397-410. https://doi.org/10.1007/ s13402-016-0281-9.

7. Zhu Z, Wang W, Lin F, Jordan T, Li G, Silverman S, et al. Genome profiles of lymphovascular breast cancer cells reveal multiple clonally differentiated outcomes with multi-regional LCM and G\&amp;T-seq. bioRxiv. 2020:807156. https://doi.org/10.1101/807156.

8. Matsuda N, Hayashi N, Ohde S, Yagata H, Kajiura Y, Yoshida A, Suzuki K, Nakamura S, Tsunoda H, Yamauchi H. A nomogram for predicting locoregional recurrence in primary breast cancer patients who received breast-conserving surgery after neoadjuvant chemotherapy. J Surg Oncol. 2014;109(8):764-9. https://doi.org/10.1002/jso.23586.

9. Ouyang FS, Guo BL, Huang XY, Ouyang LZ, Zhou CR, Zhang R, Wu ML, Yang ZS, Wu SK, Guo TD, Yang SM, Hu QG. A nomogram for individual prediction of vascular invasion in primary breast cancer. Eur J Radiol. 2019;110:30-8. https://doi.org/10.1016/j.ejrad.2018.11.013.

10. Ni-Jia-Ti MY, Ai-Hai-Ti DL, Huo-Jia AS, Wu-Mai-Er PL, A-Bu-Li-Zi AB, Shi Y, et al. Development of a risk-stratification scoring system for predicting lymphovascular invasion in breast cancer. BMC Cancer. 2020;20(1):94. https://doi.org/10.1186/s12885-020-6578-0. PMID: 32013960; PMCID: PMC6998851.

11. Cheon H, Kim HJ, Lee SM, Cho SH, Shin KM, Kim GC, Park JY, Kim WH. Preoperative MRI features associated with lymphovascular invasion in nodenegative invasive breast cancer: a propensity-matched analysis. J Magn Reson Imaging. 2017;46(4):1037-44. https://doi.org/10.1002/jmri.25710.

12. Horvat JV, Bernard-Davila B, Helbich TH, Zhang M, Morris EA, Thakur SB, Ochoa-Albiztegui RE, Leithner D, Marino MA, Baltzer PA, Clauser P, Kapetas $\mathrm{P}$, Bago-Horvath Z, Pinker K. Diffusion-weighted imaging (DWI) with apparent diffusion coefficient (ADC) mapping as a quantitative imaging biomarker for prediction of immunohistochemical receptor status, proliferation rate, and molecular subtypes of breast cancer. J Magn Reson Imaging. 2019;50(3):836-46. https://doi.org/10.1002/jmri.26697.

13. White NS, McDonald C, Farid N, Kuperman J, Karow D, Schenker-Ahmed $\mathrm{NM}$, et al. Diffusion-weighted imaging in cancer: physical foundations and applications of restriction spectrum imaging. Cancer Res. 2014;74(17):463852. https://doi.org/10.1158/0008-5472.CAN-13-3534.

14. Ren C, Zou Y, Zhang X, Li K. Diagnostic value of diffusion-weighted imaging-derived apparent diffusion coefficient and its association with histological prognostic factors in breast cancer. Oncol Lett. 2019;18(3):3295303. https://doi.org/10.3892/ol.2019.10651.

15. Martincich L, Deantoni V, Bertotto I, Redana S, Kubatzki F, Sarotto I, Rossi V, Liotti M, Ponzone R, Aglietta M, Regge D, Montemurro F. Correlations between diffusion-weighted imaging and breast cancer biomarkers. Eur Radiol. 2012;22(7):1519-28. https://doi.org/10.1007/s0033 0-012-2403-8

16. Zhao M, Fu K, Zhang L, Guo W, Wu Q, Bai X, Li Z, Guo Q, Tian J. Intravoxel incoherent motion magnetic resonance imaging for breast cancer: a comparison with benign lesions and evaluation of heterogeneity in different tumor regions with prognostic factors and molecular classification. Oncol Lett. 2018;16(4):5100-12. https://doi.org/10.3892/ol.2018.9312.

17. Karan B, Pourbagher A, Torun N. Diffusion-weighted imaging and 18Ffluorodeoxyglucose positron emission tomography/computed tomography in breast cancer: correlation of the apparent diffusion coefficient and maximum standardized uptake values with prognostic factors. J Magn Reson Imaging. 2016;43(6):1434-44. https://doi.org/10.1002/jmri.25112.

18. Mori N, Mugikura S, Takasawa C, Miyashita M, Shimauchi A, Ota H, Ishida T, kasajima A, Takase K, Kodama T, Takahashi S. Peritumoral apparent diffusion coefficients for prediction of lymphovascular invasion in clinically nodenegative invasive breast cancer. Eur Radiol. 2016;26(2):331-9. https://doi. org/10.1007/s00330-015-3847-4.

19. Yang W, Qiang JW, Tian HP, Chen B, Wang AJ, Zhao JG. Minimum apparent diffusion coefficient for predicting lymphovascular invasion in invasive cervical cancer. J Magn Reson Imaging. 2017;45(6):1771-9. https://doi.org/1 $0.1002 /$ jmri.25542

20. Choi BB. Associations between apparent diffusion coefficient values and the prognostic factors of breast cancer. J Comput Assist Tomogr. 2019;43(6): 931-6. https://doi.org/10.1097/RCT.0000000000000936.

21. Hirano M, Satake H, Ishigaki S, Ikeda M, Kawai H, Naganawa S. Diffusionweighted imaging of breast masses: comparison of diagnostic performance using various apparent diffusion coefficient parameters. AJR Am J Roentgenol. 2012;198(3):717-22. https://doi.org/10.2214/AJR.11.7093. 
22. Kang BJ, Lipson JA, Planey KR, Zackrisson S, Ikeda DM, Kao J, et al. Rim sign in breast lesions on diffusion-weighted magnetic resonance imaging: diagnostic accuracy and clinical usefulness. J Magn Reson Imaging. 2015; 41(3):616-23. https://doi.org/10.1002/jmri.24617.

23. D'Orsi CJ, Sickles EA, Mendelson EB, et al. Breast Imaging Reporting and Data System ${ }^{\oplus}$ (BI-RADS $^{\oplus}$ ) Atlas. 5th ed. Reston, VA: American College of Radiology; 2013

24. Yılmaz E, Sarı O, Yılmaz A, Ucar N, Aslan A, Inan I, et al. Diffusion-weighted imaging for the discrimination of benign and malignant breast masses; utility of ADC and relative ADC. J Belg Soc Radiol. 2018;102:24.

25. Allred DC, Harvey JM, Berardo M, Clark GM. Prognostic and predictive factors in breast cancer by immunohistochemical analysis. Mod Pathol. 1998;11(2):155-68.

26. Shen S-D, Zhong S-Z, Wang C-Z, Huang W-H. Correlation of lymphovascular invasion with clinicopathological factors in invasive breast cancer: a metaanalysis. Int J Clin Exp Med. 2015;8(10):17789-95.

27. Chan T-S, Shaked Y, Tsai KK. Targeting the interplay between cancer fibroblasts, mesenchymal stem cells, and cancer stem cells in desmoplastic cancers. Front Oncol. 2019;9. https://doi.org/10.3389/fonc.2019.00688.

28. Costantini M, Belli P, Bufi E, Asunis AM, Ferra E, Bitti GT. Association between sonographic appearances of breast cancers and their histopathologic features and biomarkers. J Clin Ultrasound. 2016;44(1):26-33. https://doi. org/10.1002/jcu.22312.

29. Dogan BE, Turnbull LW. Imaging of triple-negative breast cancer. Ann Oncol. 2012;23(Suppl 6):vi23-9.

30. Macura KJ, Ouwerkerk R, Jacobs MA, Bluemke DA. Patterns of enhancement on breast MR images: interpretation and imaging pitfalls. RadioGraphics. 2006;26(6):1719-34. https://doi.org/10.1148/rg.266065025.

31. Szabó BK, Aspelin P, Kristoffersen Wiberg M, Tot T, Boné B. Invasive breast cancer: correlation of dynamic MR features with prognostic factors. Eur Radiol. 2003;13(11):2425-35. https://doi.org/10.1007/s00330-003-2000-y.

32. Turashvili G, Brogi E. Tumor heterogeneity in breast cancer. Front Med (Lausanne). 2017:4:227.

33. Gerashchenko TS, Zavyalova MV, Denisov EV, Krakhmal NV, Pautova DN, Litviakov NV, Vtorushin SV, Cherdyntseva NV, Perelmuter VM. Intratumoral morphological heterogeneity of breast cancer as an indicator of the metastatic potential and tumor chemosensitivity. Acta Naturae. 2017;9(1): 56-67. https://doi.org/10.32607/20758251-2017-9-1-56-67.

34. Choi Y, Kim SH, Youn IK, Kang BJ, Park W-C, Lee A. Rim sign and histogram analysis of apparent diffusion coefficient values on diffusion-weighted MRI in triple-negative breast cancer: comparison with ER-positive subtype. Plos One. 2017;12:e0177903.

35. Uematsu T, Kasami M, Yuen S. Triple-negative breast cancer: correlation between MR imaging and pathologic findings. Radiology. 2009;250(3):63847. https://doi.org/10.1148/radiol.2503081054.

36. Uematsu T, Kasami M, Watanabe J. Is evaluation of the presence of prepectoral edema on T2-weighted with fat-suppression $3 \mathrm{~T}$ breast MRI a simple and readily available noninvasive technique for estimation of prognosis in patients with breast cancer? Breast Cancer. 2014;21(6):684-92. https://doi.org/10.1007/s12282-013-0440-z.

37. Bae MS, Shin SU, Ryu HS, Han W, Im SA, Park IA, Noh DY, Moon WK. Pretreatment MR imaging features of triple-negative breast cancer: association with response to neoadjuvant chemotherapy and recurrencefree survival. Radiology. 2016;281(2):392-400. https://doi.org/10.1148/radiol.2 016152331.

38. Baltzer PA, Yang F, Dietzel M, Herzog A, Simon A, Vag T, et al. Sensitivity and specificity of unilateral edema on T2W-TSE sequences in MRmammography considering 974 histologically verified lesions. Breast J. 2010; 16(3):233-9. https://doi.org/10.1111/j.1524-4741.2010.00915.x.

39. Panzironi G, Moffa G, Galati F, Marzocca F, Rizzo V, Pediconi F. Peritumoral edema as a biomarker of the aggressiveness of breast cancer: results of a retrospective study on a 3 T scanner. Breast Cancer Res Treat. 2020;181(1): 53-60. https://doi.org/10.1007/s10549-020-05592-8.

40. Cheon H, Kim HJ, Kim TH, Ryeom H-K, Lee J, Kim GC, Yuk JS, Kim WH. Invasive breast cancer: prognostic value of peritumoral edema identified at preoperative MR imaging. Radiology. 2018;287(1):68-75. https://doi.org/1 0.1148 /radiol.2017171157.

41. Okuma H, Sudah M, Kettunen T, Niukkanen A, Sutela A, Masarwah A, Kosma VM, Auvinen P, Mannermaa A, Vanninen R. Peritumor to tumor apparent diffusion coefficient ratio is associated with biologically more aggressive breast cancer features and correlates with the prognostication tools. Plos One. 2020;15(6):e0235278. https://doi.org/10.1371/journal.pone.0235278.

42. Sung JS, Jochelson MS, Brennan S, Joo S, Wen YH, Moskowitz C, Zheng J, Dershaw DD, Morris EA. MR imaging features of triple-negative breast cancers. Breast J. 2013;19(6):643-9. https://doi.org/10.1111/tbj.12182.

43. Choi BB, Lee JS, Kim KH. Association between MRI features and standardized uptake value of 18F-FDG PET/CT in triple-negative breast cancer. Oncol Res Treat. 2018;41(11):706-11. https://doi.org/10.1159/0004 92341.

44. Newell D, Nie K, Chen JH, Hsu CC, Yu HJ, Nalcioglu O, et al. Selection of diagnostic features on breast MRI to differentiate between malignant and benign lesions using computer-aided diagnosis: differences in lesions presenting as mass and non-mass-like enhancement. Eur Radiol. 2010;20(4): 771-81. https://doi.org/10.1007/s00330-009-1616-y.

45. Ayatollahi F, Shokouhi SB, Teuwen J. Differentiating benign and malignant mass and non-mass lesions in breast DCE-MRI using normalized frequencybased features. Int J Comput Assist Radiol Surg. 2020;15(2):297-307. https:// doi.org/10.1007/s11548-019-02103-Z.

46. Jansen SA, Fan X, Karczmar GS, Abe H, Schmidt RA, Giger M, et al. DCEMRI of breast lesions: is kinetic analysis equally effective for both mass and nonmass-like enhancement?. Med Phys. 2008;35(7):3102-9. https://doi.org/1 $0.1118 / 1.2936220$

\section{Publisher's Note}

Springer Nature remains neutral with regard to jurisdictional claims in published maps and institutional affiliations.
Ready to submit your research? Choose BMC and benefit from:

- fast, convenient online submission

- thorough peer review by experienced researchers in your field

- rapid publication on acceptance

- support for research data, including large and complex data types

- gold Open Access which fosters wider collaboration and increased citations

- maximum visibility for your research: over $100 \mathrm{M}$ website views per year

At BMC, research is always in progress.

Learn more biomedcentral.com/submissions 